\title{
Flexible Vehicle Scheduling Optimization with Uncertainty in Intelligent Logistic Systems
}

\author{
Lu Sun, ${ }^{1}$ Lin Lin, ${ }^{1,2,4,}{ }^{*}$ Haojie Li, ${ }^{1,4}$ and Mitsuo Gen ${ }^{2,3}$ \\ ${ }^{1}$ School of Software, Dalian University of Technology, Dalian 116600, China \\ ${ }^{2}$ Fuzzy Logic Systems Institute, Tokyo 135-0061, Japan \\ ${ }^{3}$ Tokyo University of Science, Tokyo 125-8585, Japan \\ ${ }^{4}$ Key Laboratory for Ubiquitous Network and Service Software of Liaoning Province, \\ Dalian University of Technology, Dalian 116600, China
}

(Received October 7, 2018; accepted January 28, 2019)

Keywords: vehicle scheduling, uncertain scheduling, intelligent logistic system

The flexible vehicle scheduling problem (FVSP) plays an important role in intelligent logistic systems (ILSs) as it improves transportation efficiency and reduces logistic costs through the optimization of the schedule of cargoes. FVSP is difficult to solve because it is a typical combinatorial optimization problem (COP). It has also been proved to be an NP-hard problem. In idealized models, the transportation time of cargoes in a logistic system is determined and given in advance. However, the uncertain factors in real-world logistic systems, such as traffic jams and emergencies, always lead to an uncertain transportation time. Fuzzy numbers can represent more information in real-world applications than constant or random values. Thus, in this paper, we focus on FVSP with an uncertain transportation time (uFVSP), in which the transportation time is modeled as a fuzzy number. A cooperative hybrid evolutionary algorithm (hEA) with a self-adaptive parameter mechanism is proposed and five uFVSP instances with different scales are adopted in numerical experiments to verify the effectiveness of the proposed algorithm. The results show that our proposed algorithm has better performance than other algorithms for solving uFVSP in ILSs.

\section{Introduction}

The flexible vehicle scheduling problem (FVSP) in an intelligent logistic system (ILS) has been an area of research in recent years applicable to many real-world application systems. ${ }^{(1)}$ FVSP can be treated as a typical discrete combinatorial optimization problem (COP). The objective function is minimizing the transportation cost through the optimization of the schedule of different cargoes with a set of precedence constraints. In a single ILS, various users need to transport different kinds of cargoes to their destinations in the shortest time; these cargoes have different characteristics and constraints, such as different capacities and weights. Each user has various cargoes, and different cargoes can be transported by different vehicles. An illustration of FVSP is shown in Fig. 1.

*Corresponding author: e-mail: lin@dlut.edu.cn https://doi.org/10.18494/SAM.2019.2150 


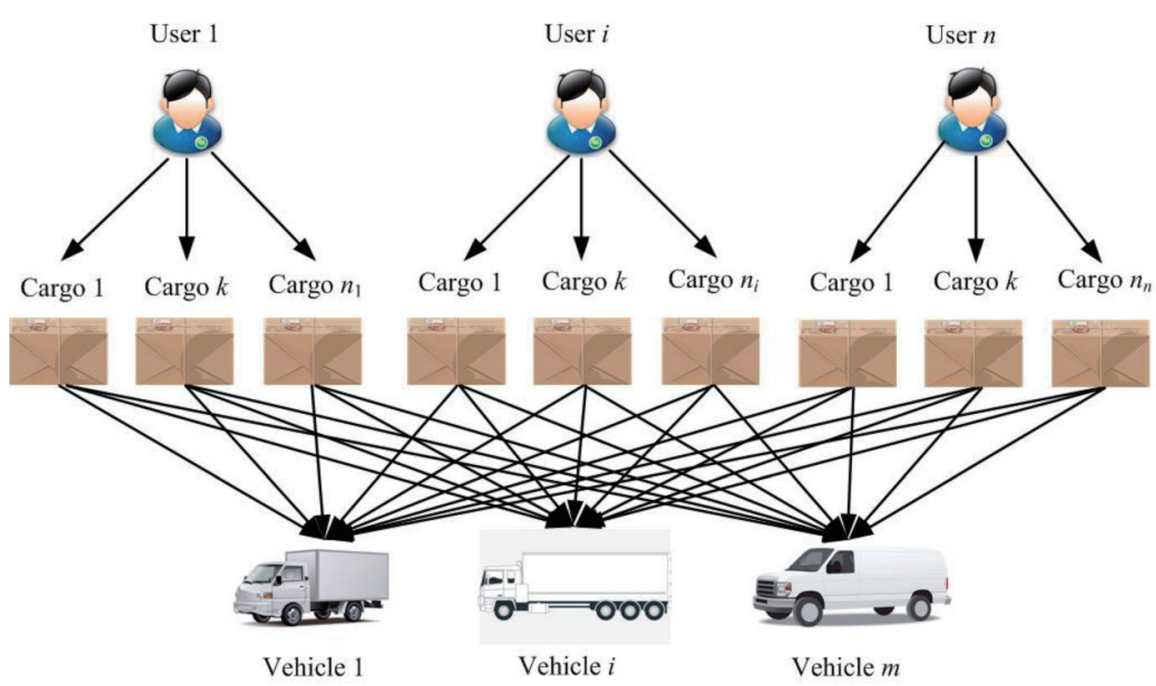

Fig. 1. (Color online) Illustration of FVSP in ILS.

\section{$1.1 \quad$ Related work}

The vehicle scheduling problem (VSP) was first studied in the 1960s and has attracted researchers' attention in recent years. ${ }^{(2)}$ It is a typical COP in a research area, and it also widely exists in many real-world application systems, such as ILS, and smart cities. ${ }^{(3)}$ With the rapid development of ILS, FVSP becomes a critical problem to solve because its flexibility can increase the efficiency of transportation and a reasonable vehicle schedule can make the use of logistic resources rational. Tarantilis et al. proposed a hybrid algorithm considering two local search strategies for VSP in logistic systems. ${ }^{(4)}$ Freling et al. considered a round-tripcondition VSP with a hybrid algorithm. ${ }^{(5)}$ Laurent and Hao ${ }^{(6)}$ and Mesquita and Paias ${ }^{(7)}$ studied a simulation algorithm for FVSP with a specific preprocessing phase to optimize the vehicle schedule. Laurent and Hao also proposed an integrated algorithm with an effective local search strategy to solve FVSP in a multiple-vehicle environment. ${ }^{(8)}$ Pepin et al. studied a hybrid algorithm combining a neighborhood search with a tabu search strategy to solve FVSP in a multiple-vehicle environment. ${ }^{(9)}$ Teng et al. proposed an effective algorithm for multiple-vehicle resource VSPs. ${ }^{(10)}$

In practice, there are always uncertain factors in real-world ILSs, such as traffic jams, which lead to the variables in the speed from one moment to another. Therefore, the transportation cost cannot be modeled by a single value or a determined value unlike in ideal and traditional FVSPs. Recently, researchers have focused on FVSP with an uncertain transportation time (uFVSP) for both theoretical research and practical analysis. Huisman et al. proposed a hybrid heuristics algorithm for FVSP in an uncertain environment. ${ }^{(11)}$ Hadiar and Soumis studied an algorithm with a comprehensive branch and price strategy for FVSP with uncertain time windows. ${ }^{(12)}$ Wei et al. proposed an effective algorithm with an organic fusion optimization strategy for FVSP with a fuzzy transportation cost. ${ }^{(13)}$ Shen et al. studied an effective probabilistic algorithm for uFVSP. $^{(14)}$ 
Evolutionary algorithms (EAs) adopt and apply the principles and rules of biology and genetics to find the optimal solution through suitable encoding and decoding mechanisms. ${ }^{(15)}$ EAs are highly efficient for searching the optimal solution to problems that cannot be solved by traditional algorithms, including NP-hard problems, COPs, and high-dimensional optimization problems. As discussed above, FVSP is a typical COP and has been proved to be an NP-hard problem. $^{(16)}$ Therefore, EAs are popular and have been widely used to solve FVSP in recent years. ${ }^{(17)}$ The application of EAs to FVSP can be classified into two cases, i.e., FVSP and uFVSP. For FVSP, Zuo et al. proposed a novel heuristics algorithm with the help of Pareto solutions for VSP with a determined transportation cost. ${ }^{(18)}$ Zheng et al. researched an improved genetic algorithm with a self-adaptive strategy and a novel update strategy that used a quantum rotation angle for VSP. ${ }^{(19)}$ In addition to EAs for solving FVSP in the determined environment, there are also EAs for solving uFVSP. Gu et al. proposed an improved cooperative EA for solving scheduling problems in which the processing time is modeled by stochastic values. ${ }^{(20)}$ Zhang et al. studied an effective EA that combined GA and particle swarm optimization (PSO) for FVSP with bounded processing times and transportation constraints. ${ }^{(21)}$

\subsection{Motivation and contributions}

As discussed above, although various algorithms have been proposed for solving FVSP, they cannot be directly applied to uFVSP. Thus, we have proposed a hybrid evolutionary algorithm (hEA) combining GA and PSO with novel encoding and decoding strategies and a local search strategy. The contributions of this paper are listed as follows:

(a) One-section encoding and decoding strategies are designed and applied to both GA and PSO, adopting a random key representation. The decimal and integer parts represent the cargo sequence and vehicle assignment, respectively.

(b) A new population initialization is designed and adopted in the hEA. Each gene is initialized according to the given upper and lower bounds to avoid searching in an invalid solution space.

(c) A cooperative coevolutionary framework is improved and an improved coevolutionary framework is adopted in our proposed algorithm. The aim is to search for the optimal solution in multiple subsolution spaces instead of the size of the whole solution space because the whole solution space increases exponentially with the scale of the problem, reducing the performance degradation of the proposed algorithm.

This paper is organized as follows. The formulation of UFVSP is introduced in Sect. 2, the proposed hEA is designed and proposed in Sect. 3. Numerical experiments and results are described in Sect. 4. The final conclusion is presented in Sect. 5.

\section{Mathematical Model of uFVSP}

In this section, we model the transportation cost using fuzzy numbers instead of single integer values. FVSP can be described as an extension problem of VSP. Each cargo can be transported by any available vehicle. There exist precedent constraints among cargoes of the 
same user. Each cargo $C_{i k}$ can be transported by an available vehicle $V_{j}$ with a transportation cost modeled by a triangular fuzzy number (TFN) $T_{i k j}=\left(t_{i k j}^{1}, t_{i k j}^{2}, t_{i k j}^{3}\right)$, where $t_{i k j}^{1}, t_{i k j}^{2}$, and $t_{i k j}^{3}$ stand for the minimum, maximum-likelihood, and maximum values, respectively. The exact transportation cost cannot be estimated before the completion of transportation. Therefore, we construct various concept scenarios $\zeta$ that correspond to the processing time. The objective of solving uFVSP is to minimize the maximum fuzzy transportation time $\left(T_{\max }\right)$ of all users.

\section{Indices:}

$$
\begin{array}{ll}
i & \text { user ID }(i=1, \ldots, n) \\
k & \text { cargo ID }\left(k=1, \ldots, n_{i}\right) \\
j & \text { vehicle ID }(j=1, \ldots, m)
\end{array}
$$

\section{Parameters:}
$n \quad$ total number of users
$m \quad$ total number of vehicles
$n_{i} \quad$ total number of cargoes of user $i$
$U_{i} \quad i$ th user
$C_{i k} \quad k$ th cargo of user $i$
$V_{j} \quad j$ th vehicle
$T_{i k j} \quad$ fuzzy transportation cost of $C_{i k}$ by $V_{j}$

\section{Decision variables:}

$x_{i k j} \quad 1$ stands for $C_{i k}$ transported by $V_{j}$ 0 stands for $C_{i k}$ not transported by $V_{j}$

$T_{i k j}^{S}, T_{i k j}^{T} \geq 0$, start and end times of $C_{i k}$ transported by $V_{j}$

The formulation of uFVSP is written as

$$
\min E\left[T_{\max }\right]=\max _{i}\left\{\max _{k}\left\{\max _{j} E\left[T_{i k j}^{T}\right]\right\}\right\}
$$

where $T_{i k j}^{T}=T_{i k j}^{S}+T_{i k j}$

s.t.

$$
\begin{gathered}
\sum_{j=1}^{m} x_{i k j} \leq 1, \forall i, k, \\
T_{i k j}^{S}-T_{i k^{\prime} j}^{T} \geq 0, \forall i,\left(k \succ k^{\prime}\right), \\
\left(\sum_{i=1}^{n} \sum_{k=1}^{n_{i}} x_{i k j} \cdot T_{i k j}^{S} \leq \sum_{i=1}^{n} \sum_{k^{\prime}=1}^{n_{i}} x_{i k^{\prime} j} \cdot T_{i k^{\prime} j}^{S}\right) \wedge\left(\sum_{i=1}^{n} \sum_{k=1}^{n_{i}} x_{i k j} \cdot T_{i k j}^{T} \leq \sum_{i=1}^{n} \sum_{k^{\prime}=1}^{n_{i}} x_{i k^{\prime} j} \cdot T_{i k^{\prime} j}^{S}\right), \forall i, \\
x_{i k j} \in\{0,1\}, \forall i, k, j, \\
T_{i k j}^{S}, T_{i k j}^{T} \geq 0, \forall i, k, j .
\end{gathered}
$$


Here, Eq. (1) is the objective function of uFVSP. Equation (2) ensures that each cargo is transported only once. Equations (3) and (4) ensure the precedence constraint of the cargoes of identical users. The successor cargo needs to be transported after the completion of the preorder cargo of the same user. Equations (5) and (6) represent nonnegativity restrictions.

\section{Proposed Algorithm}

In this section, the details of our proposed hEA, i.e., representation, evolutionary operators, and parameter self-adaptation, are introduced and described. A flow chart of our proposed hEA is shown in Fig. 2.

\subsection{Representation}

Representation plays an important role in EAs because a suitable representation can help EA search in a more meaningful solution space. The proposed hEA adopts a two-section representation for both GA and PSO. The two sections represent the cargo sequence and vehicle assignment. As shown in Fig. 3, the total length of each individual is twice the total number

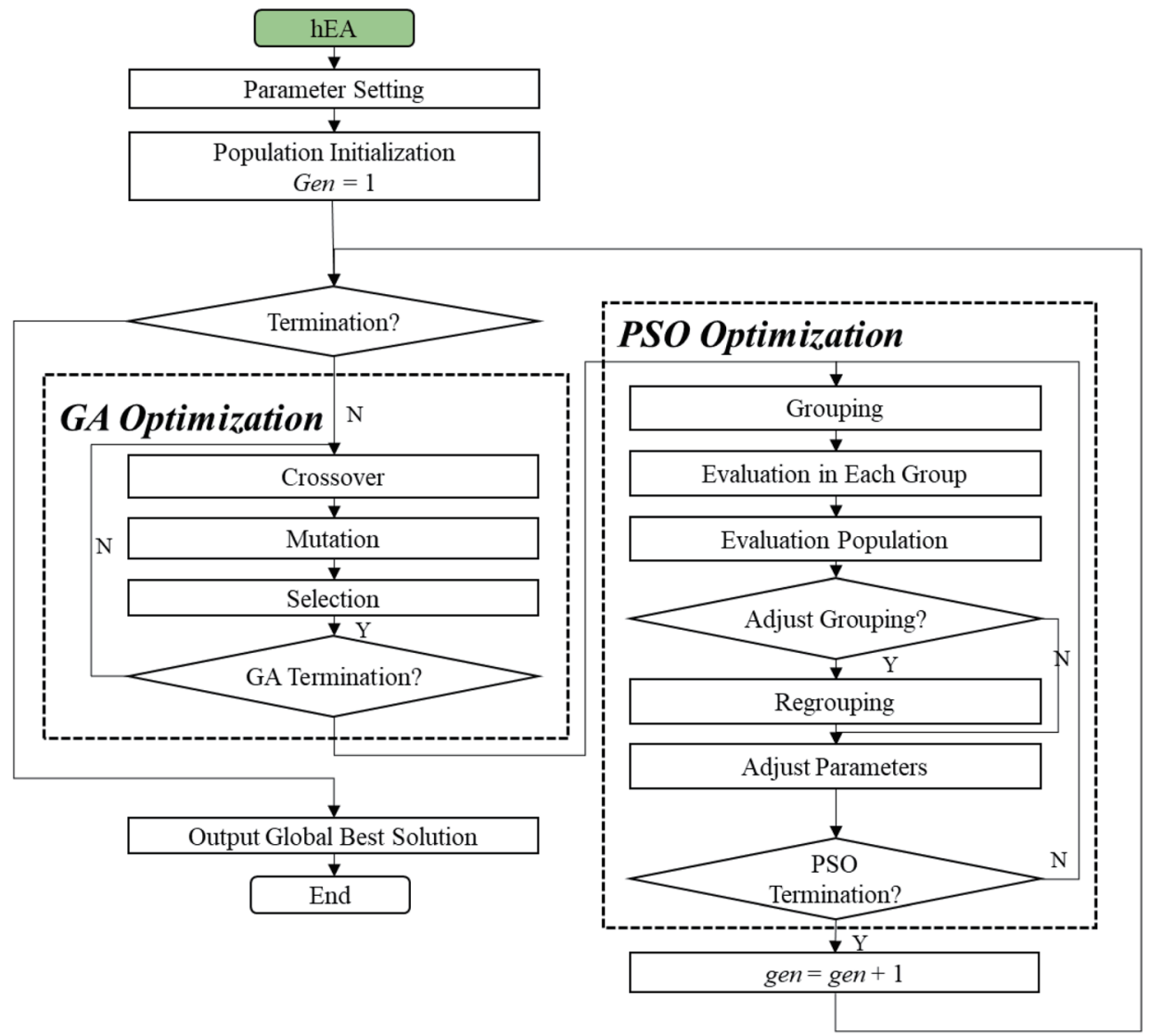

Fig. 2. (Color online) Flow chart of the proposed hEA. 


$$
\begin{array}{|l|l|l|l|l|l|l|l|}
C_{11} & C_{12} & C_{13} & C_{21} & C_{22} & C_{31} & C_{32} \\
\hline 3.1 & 1.8 & 1.2 & 2.7 & 1.5 & 2.7 & 2.3 \\
\hline
\end{array}
$$

(a)

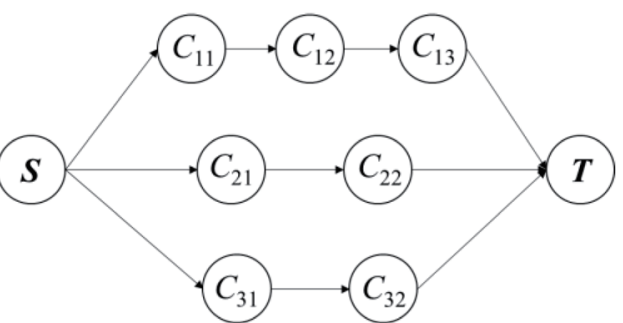

(b)

Fig. 3. Illustration of (a) random key representation and (b) network structure used for decoding.

of all cargoes. Then, we decode the individuals through a priority-based rule with the help of a network decoding mechanism, which ensures that the decoded solutions are all feasible solutions. ${ }^{(22)}$ This rule excludes more than $90 \%$ of infeasible solutions to increase the search efficiency of the proposed hEA. For the second section, each real number must be rounded to an integer; the integer represents the index of the vehicle assigned to the corresponding cargo.

\subsection{Evolutionary operators}

(1) Genetic operators:

The proposed hEA adopts a probability-based crossover strategy (P-crossover). Two chromosomes are selected, one of which is selected from the top of a list of chromosomes listed in decreasing order of their fitness. Then, the proposed hEA generates a set of random real values from $[0,1]$, and the size of this set is equal to the length of the chromosome. We set the crossover probability as 0.7 . As shown in Fig. 4, if the random value is larger than 0.7, the gene of the second individual is inherited by the offspring. If the random value is smaller than 0.7, the gene of the first individual is inherited by the offspring. The selection mechanism used in the hEA is tournament selection. ${ }^{(22)}$ The individuals with better performance are selected in the next generation.

(2) PSO update strategy:

The PSO update strategy adopted in this paper is expressed as

$$
v_{i}(q+1)=\left\{\begin{array}{l}
w v_{i}(q)+c_{1} r_{1}\left[p_{\text {best }}(q)-x_{i}(q)\right]+c_{2} r_{2}\left[g_{\text {best }}(q)-x_{i}(q)\right], \text { rand }(0,1) \geq p \\
w v_{i}(q)+c_{1} r_{1}\left[l_{\text {best }}(q)-x_{i}(q)\right]+c_{2} r_{2}\left[g_{\text {best }}(q)-x_{i}(q)\right], \text { otherwise }
\end{array}\right.
$$

where $w$ represents the inertia weight used to determine the percentage of the current velocity. $c_{1}$ and $c_{2}$ are constants, and $r_{1}$ and $r_{2}$ are two random values from [0,1]. $v_{i}(q)$ and $x_{i}(q)$ represent the velocity and position of an individual in the qth generation, respectively. $p_{\text {best }}(q), l_{\text {best }}(q)$, and $g_{\text {best }}(q)$ represent the personal best, local best, and global best positions of an individual in the $q$ th generation, respectively. This update equation performs well in balancing exploration and exploitation, which helps the hEA find an optimal solution. 


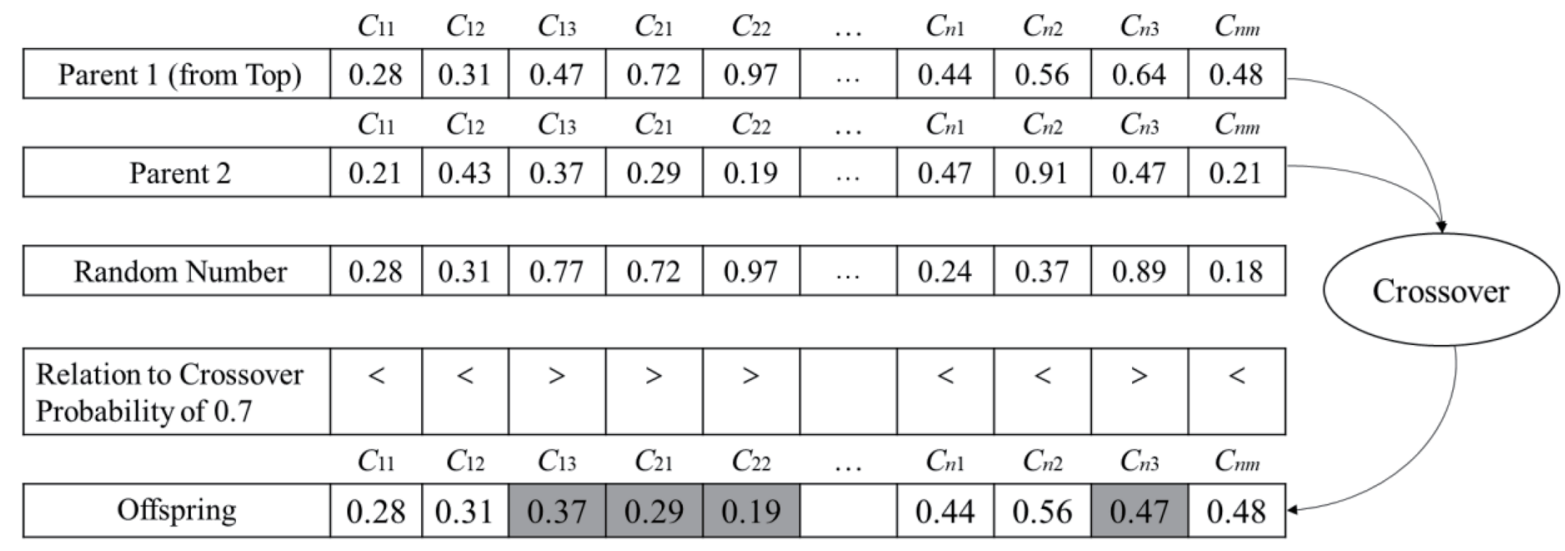

Fig. 4. Illustration of crossover.

\subsection{Self-adaptation of parameters}

As mentioned above, $\mathrm{w}$ is a key parameter, which is adjusted using the equation

$$
w= \begin{cases}N(0.5,0.3), & \text { if } U(0,1)<f p \\ C(0.1), & \text { otherwise }\end{cases}
$$

where $N, U$, and $C$ represent normal distributions with parameters of 0.5 and 0.3 and a Cauchy distribution with a parameter of 0.1 , respectively. $f p$ represents the selection probability. We record the four parameters used for the self-adjustment of the selection probability, i.e., $s n_{1}, s n_{2}$, $f n_{1}$, and $f n_{2}$, after each 20 iterations. They represent the numbers of offspring generated by $p_{\text {best }}$ and $l_{\text {best }}$ that are successfully and unsuccessfully propagated to the next iteration, respectively. All four values are counted and recorded. The update equation is written as

$$
f p=\frac{s n_{1}\left(s n_{2}+s f_{2}\right)}{s n_{2}\left(s n_{1}+s f_{1}\right)+s n_{1}\left(s n_{2}+s f_{2}\right)} .
$$

\section{Numerical Experiments and Analysis}

In this section, the experimental settings and analysis employed to verify the effectiveness of the hEA are introduced. The proposed algorithm and all the algorithms used for comparison are implemented on a PC with an Intel(R) Core(TM) i7-4790 CPU @3.60 GHz and 12 GB RAM. Five fuzzy instances are adopted to verify the superiority of the proposed hEA. Three typical EAs, i.e., the $\mathrm{GA},{ }^{(23)} \mathrm{PSO},{ }^{(24)}$ and differential evolution (DE) ${ }^{(25)}$ are those used for comparison with the proposed hEA. The parameters used in this section and those used for various EAs are listed in Tables 1 and 2, respectively. 
Table 1

Parameter settings.

\begin{tabular}{lccccc}
\hline Instance & Pop. Size & Group Size & hGA Gens & GA Gens & PSO Gens \\
\hline uFVSP 1 & 100 & 100 & 1000 & 50 & 50 \\
uFVSP 2 & 100 & 100 & 1000 & 50 & 50 \\
uFVSP 3 & 100 & 100 & 1000 & 50 & 50 \\
uFVSP 4 & 100 & 100 & 1000 & 50 & 50 \\
uFVSP 5 & 100 & 100 & 1000 & 50 & 50 \\
\hline
\end{tabular}

Table 2

Parameter settings of algorithms.

\begin{tabular}{lccccccc}
\hline Alg. & $c_{1}$ & $c_{2}$ & $w$ & Cr.Prob & Mu.Prob & $F$ & $C$ \\
\hline GA & - & - & - & 0.5 & 0.5 & - & - \\
PSO & 1.49 & 1.49 & 0.5 & - & - & - & - \\
DE & - & - & - & - & - & 1 & 0.5 \\
hEA & 1.49 & 1.49 & 0.5 & 0.5 & 0.5 & - & - \\
\hline
\end{tabular}

\subsection{Description of fuzzy instances in FVSP}

Five fuzzy instances are adopted in this section, and they are named uFVSP1-uFVSP5. The number of users ranges from 10 to 15 and the total number of cargoes ranges from 40 to 80 . The total number of available vehicles ranges from 10 to 15 . To avoid anomalous experimental results, all experiments are run 20 times and the calculated average values are given as the final experimental results.

\subsection{Superiority of hEA}

In this subsection, three typical EAs, i.e., GA, PSO, and DE, are adopted for comparison with the hEA to verify the effectiveness of the hEA. We record three criteria, i.e,. the best fuzzy transportation cost (Best), the average fuzzy transportation cost (Average), and the worst fuzzy transportation cost (Worst), to show the superiority and stability of the hEA. The results are listed in Table 3. Our proposed hEA performs better for all five instances. This is because the hybrid of different EAs can balance the advantages and disadvantages of each EA. The proposed hEA can balance the ability of exploration and exploitation so that the hEA can search for better solutions. To remove the effect caused by the local search strategy, Table 4 lists the results of three typical EAs considering the same local search strategy in the hEA. The results show that our proposed hEA still has the best performance.

\subsection{Superiority of self-adaption of parameters}

In this section, we give the numerical experiments for the superiority of self-adaption of parameters. We compare the performance of the hEA with that of the hEA without a parameter self-adaptive strategy (marked hEA' for short) in four instances, i.e., uFVSP1-uFVSP4. The 
Table 3

Performance characteristics of hEA compared with those of three typical EAs.

\begin{tabular}{lcccc}
\hline Instance & GA & PSO & DE & hEA \\
\hline uFVSP1-Best & $(33,47,62)$ & $(31,45,60)$ & $(40,55,74)$ & $(\mathbf{2 1 , 3 0 , 4 4 )}$ \\
Average & $(37.0,52.3,68.0)$ & $(37.2,50.6,63.7)$ & $(40.1,56.3,73.4)$ & $(\mathbf{2 2 . 2 , 3 1 . 1 , 4 3 . 2})$ \\
Worst & $(42,55,70)$ & $(41,55,70)$ & $(41,55,70)$ & $\mathbf{( 3 5 , 3 2 , 4 3 )}$ \\
uFVSP2-Best & $(53,72,92)$ & $(52,70,94)$ & $(52,73,93)$ & $\mathbf{( 4 4 , 6 1 , 7 6 )}$ \\
Average & $(56.6,82.8,100.3)$ & $(54.8,64.9,99.0)$ & $(58.3,80.7,104.2)$ & $\mathbf{( 4 3 . 1 , 6 3 . 6 , 8 4 . 2 )}$ \\
Worst & $(64,82,107)$ & $(64,82,109)$ & $(66,89,117)$ & $\mathbf{( 5 0 , 7 2 , 9 4 )}$ \\
uFVSP3-Best & $(55,80,101)$ & $(56,77,95)$ & $(57,81,104)$ & $\mathbf{( 3 1 , 5 0 , 6 6 )}$ \\
Average & $(59.6,81.7,106.1)$ & $(56.7,78.3,100.7)$ & $(60.3,94.4,108.2)$ & $\mathbf{( 3 5 . 2 , 4 8 . 1 , 6 3 . 7 )}$ \\
Worst & $(61,84,105)$ & $(30.7,43.3,60.6)$ & $(61,88,114)$ & $\mathbf{( 3 8 , 4 9 , 6 1 )}$ \\
uFVSP4-Best & $(44,60,82)$ & $(44,60,83)$ & $(44,61,82)$ & $\mathbf{( 2 9 , 3 7 , 5 3 )}$ \\
Average & $(45.4,63.3,87.3)$ & $(45.5,63.6,87.3)$ & $(44.5,63.3,87.3)$ & $\mathbf{( 2 7 . 3 , 3 9 . 4 , 5 3 . 7 )}$ \\
Worst & $(47,67,93)$ & $(47,68,92)$ & $(48,70,95)$ & $\mathbf{( 2 6 , 4 0 , 5 5 )}$ \\
uFVSP5-Best & $(67,98,131)$ & $(68,96,130)$ & $(67,102,134)$ & $\mathbf{( 4 2 , 5 7 , 8 1 )}$ \\
Average & $(70.3,101.7,133.4)$ & $(70.1,101.2,133.3)$ & $(70.3,102.7,133.4)$ & $\mathbf{( 4 5 . 8 , 6 3 . 2 , 8 1 . 8})$ \\
Worst & $(73,106,136)$ & $(74,105,136)$ & $(74,113,148)$ & $\mathbf{( 4 7 , 6 5 , 8 3 )}$ \\
\hline
\end{tabular}

Table 4

Performance characteristics of hEA compared with those of three typical EAs.

\begin{tabular}{lcccc}
\hline Instance & GA+LS & PSO+LS & DE+LS & hEA \\
\hline uFVSP1-Best & $(22,32,41)$ & $(21,42,43)$ & $(24,42,43)$ & $(\mathbf{2 1 , 3 0 , 4 4 )}$ \\
Average & $(23.1,31.7,44.7)$ & $(22.8,31.7,45.3)$ & $(23.6,33.2,44.8)$ & $\mathbf{( 2 2 . 2 , 3 1 . 1 , 4 3 . 2 )}$ \\
Worst & $(25,33,41)$ & $(20,34,48)$ & $(20,34,48)$ & $\mathbf{( 3 5 , 3 2 , 4 3 )}$ \\
uFVSP2-Best & $(41,58,78)$ & $(41,59,78)$ & $(42,64,84)$ & $\mathbf{( 4 4 , 6 1 , 7 6 )}$ \\
Average & $(47.1,54.7,82.1)$ & $(46.8,64.1,85.4)$ & $(43.7,63.2,86.2)$ & $\mathbf{( 4 5 . 1 , 6 6 . 6 , 8 6 . 2 )}$ \\
Worst & $(53,72,93)$ & $(51,75,97)$ & $(47,60,72)$ & $\mathbf{( 5 0 , 7 2 , 9 4 )}$ \\
uFVSP3-Best & $(34,47,64)$ & $(34,48,63)$ & $(37,47,69)$ & $\mathbf{( 3 1 , 5 0 , 6 6 )}$ \\
Average & $(35.0,48.4,63.2)$ & $(35.1,48.2,63.7)$ & $(35.3,49.3,65.1)$ & $\mathbf{( 3 5 . 2 , 4 8 . 1 , 6 3 . 7 )}$ \\
Worst & $(34,49,67)$ & $(36,52,70)$ & $(32,51,65)$ & $\mathbf{( 3 8 , 4 9 , 6 1 )}$ \\
uFVSP4-Best & $(29,37,43)$ & $(29,37,51)$ & $(28,40,53)$ & $\mathbf{( 2 9 , 3 7 , 5 3 )}$ \\
Average & $(28.3,41.2,56.0)$ & $(28.6,42.2,57.7)$ & $(27.3,39.5,54.5)$ & $\mathbf{( 2 7 . 3 , 3 9 . 4 , 5 3 . 7 )}$ \\
Worst & $(25,44,65)$ & $(26,44,64)$ & $(31,42,53)$ & $\mathbf{( 2 4 , 4 0 , 5 5 )}$ \\
uFVSP5-Best & $(43,66,85)$ & $(47,62,84)$ & $(44,63,85)$ & $\mathbf{( 4 2 , 5 7 , 8 1 )}$ \\
Average & $(47.6,66.3,88.1)$ & $(45.1,65.3,87.5)$ & $(45.2,65.1,85.3)$ & $\mathbf{( 4 5 . 8 , 6 3 . 2 , 8 1 . 8 )}$ \\
Worst & $(51,67,82)$ & $(41,64,89)$ & $(36,65,83)$ & $\mathbf{( 4 7 , 6 5 , 8 3 )}$ \\
\hline
\end{tabular}

Table 5

Performance of the hEA and the hEA without self-adaption of parameters.

\begin{tabular}{lccccc}
\hline Instance & hEA $^{\prime}$ & hEA & Instance & hEA' & hEA \\
\hline uFVSP1-Best & $(24,34,42)$ & $\mathbf{( 2 1 , 3 0 , 4 4 )}$ & uFVSP2-Best & $(42,59,80)$ & $\mathbf{( 4 4 , 6 1 , 7 6 )}$ \\
Worst & $(28,35,44)$ & $\mathbf{( 3 5 , 3 2 , 4 3 )}$ & Worst & $(54,73,95)$ & $\mathbf{( 5 0 , 7 2 , 9 4 )}$ \\
uFVSP3-Best & $(35,46,65)$ & $\mathbf{( 3 1 , 5 0 , 6 6 )}$ & uFVSP4-Best & $(33,39,46)$ & $\mathbf{( 2 9 , 3 7 , 5 3 )}$ \\
Worst & $(36,51,68)$ & $\mathbf{( 3 8 , 4 9 , 6 1 )}$ & Worst & $(27,43,58)$ & $\mathbf{( 2 4 , 4 0 , 5 5 )}$ \\
\hline
\end{tabular}

Best and Worst performance characteristics are recorded in Table 5. We can see that the hEA performs better than the hEA' in all four instances. The reason for this is that the parameter self-adaptive strategy can adjust the parameters on the basis of the performance and fitness during optimization. The adjusted parameters certainly perform better than the determined parameters given in advance when facing uncertain factors. 


\section{Conclusion}

The optimization of FVSP has significant meaning for both research-area and real-world ILSs. However, the uncertainty existing in the real world cannot be ignored. Therefore, in this paper, we consider FVSP with uncertain factors, in which the transportation cost is modeled by a TFN, and the objective is to minimize the maximum fuzzy transportation cost of all users. We propose a hybrid EA combining GA and PSO to balance the ability of local search and global search. Then, a cooperative co-evolutionary framework is imported in order to help the hEA search for the optimal solution in multiple subsolution spaces to increase the probability of finding the optimal solution. To balance uncertain factors, a self-adaptive parameter mechanism is also proposed. Five fuzzy instances are adopted and the results of numerical experiments demonstrate the superiority of the hEA.

\section{Acknowledgments}

This work is partly supported by the National Natural Science Foundation of China under Grant 61572100 and in part by a Grant-in-Aid for Scientific Research (C) of Japan Society of Promotion of Science (JSPS) No. 15K00357.

\section{References}

1 A. Adamski: Procedia-Social Behav. Sci. 20 (2011) 1004. https://doi.org/10.1016/j.sbspro.2011.08.109

2 M. M. Webber: Eur. J. Oper. Res. 97 (1997) 3. https://doi.org/10.1016/S0377-2217(96)00298-6

3 V. Pillac, M. Gendreau, C. Guret, and A. L. Medaglia: Eur. J. Oper. Res. 225 (2013) 1. https://doi.org/10.1016/ j.ejor.2012.08.015

4 C. D. Tarantilis, E. E. Zachariadis, and C. T. Kiranoudis: IEEE Trans. Intell. Transport. Sys. 10 (2009) 2. https://doi.org/10.1109/TITS.2009.2020187

5 R. Freling, D. Huisman, and A. P. M. Wagelmans: Proc. Computer-aided Scheduling of Public Transport (Springer, London, 2012) 73-90.

6 B. Laurent and J. K. Hao:. Comput. Ind. Eng. 53 (2007) 542. https://doi.org/10.1109/TITS.2009.2020187

7 M. Mesquita and A. Paias: Comput. Oper. Res. 35 (2008) 1562. https://doi.org/10.1016/j.cor.2006.09.001

8 B. Laurent and J. K. Hao: Comput. Ind. Eng. 57 (2009) 277. https://doi.org/10.1016/j.cie.2008.11.028

9 A.-S. Pepin, G. Desaulniers, A. Hertz, and D. Huisman: J. Schedul. 12 (2009) 1. https://doi.org/10.1007/s10951008-0072-x

10 J. Teng, S. Jin, and X. Lai: Math. Prob. Eng. 2015 (2015) 1. https://doi.org/10.1155/2015/506794

11 D. Huisman, R. Freling, and A. P. M. Wagelmans: Trans. Sci. 38 (2014) 4. https://doi/abs/10.1287/trsc.1030.006

12 A. Hadiar and F. Soumis: Comput. Oper. Res. 36 (2009) 7. https://doi.org/10.1016/j.cor.2008.08.010

13 M. Wei, X. Chen, and B. Sun: J. Intell. Fuzzy Sys. 29 (2015) 6. https://doi.org/10.3233/IFS-151972

14 Y. Shen, J. Xu, and J. Li: Trans. Res. B 85 (2016) 19. https://doi.org/10.1016/j.trb.2015.12.016

15 M. Gen and L. Lin: J. Intell. Manuf. 25 (2014) 849. https://doi.org/10.1007/s10845-013-0804-4

16 Y. Jin and J. Branke: IEEE Trans. Evol. Comput. 9 (2005) 3. https://doi.org/10.1109/TEVC.2005.846356

17 M. Gen, R. Cheng, and L. Lin: Network Models and Optimization: Multiobjective Genetic Algorithm Approach (Springer, London, 2008) p. 105.

18 X. Zuo, C. Chen, W. Tan, and M. Zhou: IEEE Trans. Intell. Trans. 16 (2015) 2. https://oi.org/10.1109/ TITS.2014.2352599

19 D. Zheng, J. Mao, N. Guo, C. Wang, and W. Qu: Proc. Control and Decision Conf. (Springer, Las Vegas, 2016) $2147-2150$.

20 J. Gu, M. Gu, C. Cao, and X. Gu: Comput. Oper. Res. 37 (2010) 927. https://doi.org/10.1016/j.cor.2009.07.002

21 Q. Zhang, H. Manier, and M. Manier: Comput. Oper. Res. 39 (2012) 7. https://doi.org/10.1016/j.cor.2011.10.007

22 X. Li and X. Yao: IEEE Trans. Evolut. Comput. 16 (2012) 210. https://doi.org/10.1109/TEVC.2011.2112662 
23 F. Pezzella, G. Morganti, and G. Ciaschetti: Comput. Oper. Res. 35 (2008) 10. https://doi.org/10.1016/ j.cor.2007.02.014

24 J. Kennedy: Encyclopedia of Machine Learning: Particle Swarm Optimization (Springer, London, 2011) pp. 760-766.

25 K. Price, R. M. Storn, and J. A. Lampinen: Differential Evolution: A Practical Approach to Global Optimization (Springer, London, 2006) p. 145.

\section{About the Authors}

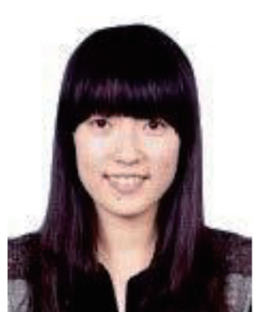

Lu Sun received her B.S. and M.S. degrees from Dalian University of Technology in 2013 and 2015, respectively, and is currently a Ph.D. student at the School of Software, Dalian University of Technology. She focuses on evolutionary algorithm, probabilistic graphical models, and their applications in combinatorial optimization problems. (sunlu517@mail.dlut.edu.cn)

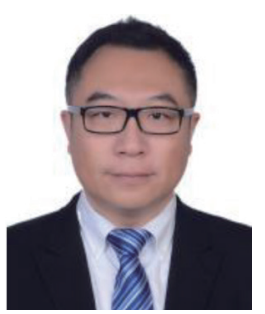

Lin Lin is currently an associate professor at the School of Software, Dalian University of Technology (DLUT), China and is a senior researcher at Fuzzy Logic Systems Institute, Japan. He received his M.Sc and Ph.D. degrees in engineering from Graduate School of Information, Production and Systems, Waseda University, in 2005 and 2008, respectively. He was a research assistant at the Information, Production and Systems Research Center (IPSRC), Waseda University, from April 2006 to March 2008, a visiting lecturer at IPSRC, Waseda University, and a postdoctoral research associate supported by the Kitakyushu Foundation for the Advancement of Industry, Science and Technology (FAIS). His core research interests are computational intelligence, deep learning, probabilistic graphical models, and their applications in combinatorial optimization and pattern recognition.

(lin@dlut.edu.cn)

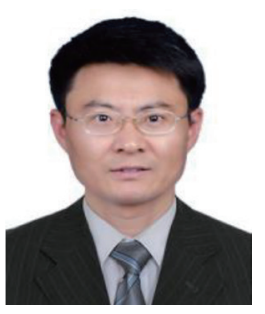

Haojie Li received his B.E. degree from Nankai University, Tianjin, China, in 1996 and his Ph.D. degree from the Institute of Computing Technology, Chinese Academy of Sciences, Beijing, China, in 2007. He is a professor at the School of Software, Dalian University of Technology, Dalian, China. From 2007 to 2009, he was a research fellow at the School of Computing, National University of Singapore, Singapore. He has coauthored more than 50 journal and conference papers, including those submitted to IEEE Transaction on Circuits and Systems for Video Technology, IEEE Transactions on Multimedia, IEEE Transactions on Image Processing, ACM Multimedia, and ACM ICMR. His research interests include social media computing, multimedia information retrieval, and probability graph models. Professor Li is a member of ACM and IEEE. (hjli@dlut.edu.cn) 


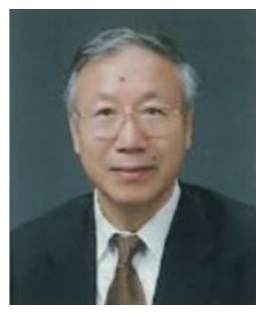

Mitsuo Gen received his B.E., M.E., and Ph.D. degrees in electronic engineering from Kogakuin University in 1969, 1971, and 1975, respectively and his Ph.D. degree in informatics from Kyoto University, Japan, in 2006. $\mathrm{He}$ is a senior research scientist at Fuzzy Logic Systems Institute and a visiting professor at Tokyo University of Science, Japan. He was a professor in the Graduate School of Information, Production and Systems, Waseda University, Japan from 2003 to 2010. He was a visiting professor at the National Tsinghua University in Taiwan from 2012 to 2014, Department of Industrial and Management Engineering, a Hanyang chair professor of Hanyang University in Korea from 2010 to 2012, and a visiting professor in the Department of IE \& OR, the University of California, Berkeley, CA from 1999 to 2000 and in the Department of Industrial Engineering, Texas A\&M University in College Station, TX, in 2000. He has coauthored five books such as Introduction to Evolutionary Algorithms (Springer in 2010), Network Models and Optimization: Multiobjective Genetic Algorithm Approach (Springer in 2008), and Genetic Algorithms \& Engineering Optimization. $\mathrm{He}$ is an area editor of Computers \& Industrial Engineering and an editorial board member of several international journals. (gen@flsi.or.jp) 